\title{
Design of Network Control System for Car Lights based on CAN Bus ZHENG Wei ${ }^{1,}$, , HAN Guangsheng ${ }^{2, b}$ \\ ${ }^{1,2}$ China Automotive Technology \& Research Center, Tianjin, China \\ a zhengwei@catarc.ac.cn, ${ }^{b}$ hanguangsheng@catarc.ac.cn
}

Keywords: CAN bus; car lights; network control system.

\begin{abstract}
Aiming at the requirements of reducing the wires and cost of vehicular electrical systems, it is presented a scheme of the network control system for car lights. It is firstly introduced the characteristics of Controller Area Network (CAN) bus which is generally implemented in control systems for modern car lights. Moreover, the hardware design processes related to the network control system for car lights is elaborated in this paper.
\end{abstract}

\section{Introduction}

With the demands of safety and comfortableness for modern vehicles, electrical/electronic technologies and their related equipment have been widely used in the vehicle body. When enjoying the conveniences brought by the equipment, we have to endure the lower reliability and maintainability due to extended wires, insufficient space and complex wiring problems.

In order to solve these problems, some automakers and automotive suppliers apply the network technologies such as CAN, LIN and SAE J1939 etc. for managing the vehicular electrical/electronic equipment nowadays. Among these in-vehicle network technologies, CAN bus is the most popular one. CAN bus protocol was firstly presented by Robert BOSCH GmbH in 1980s. It transmits signals and exchanges data among all these equipment with a pair of wires. That's to say, vehicular electrical/electronic equipment can communicate to each other over the pair of wires. Therefore, all the vehicle information can be shared easily and effectively. It is also reduced the wires in the vehicle body.

\section{Characteristics of Controller Area Network}

Controller Area Network (CAN) is a type of serial communication protocol which could effectively complete the framing of the data communication. Comparing common field buses, it supports the data communication with the higher reliability and real-time capability due to its own characteristics as follows:

1) The communication media of CAN bus could be the coaxial cable, the twisted-pair or the fiber-optic;

2) It supports multi-master style of network communication, i.e. any node in the CAN bus network could actively send messages at any time;

3) The communication distance is up to $10 \mathrm{~km}$ and the data transmit rate is up to $1 \mathrm{Mbit} / \mathrm{s}$ (when the communication distance is approximately $40 \mathrm{~m}$ );

4) Any node on the CAN bus network could be assigned different level of priorities to meet the demand of different real-time capabilities;

5) Any node on the CAN bus network could accomplish point to point, multicast or broadcast communication modes through filtering the message identifiers;

6) Each CAN frame has CRC checksum and other error correction measures to guarantee the highly reliability of data transmission, especially in the high interference environment;

7) Adopting short frame structure which means that each frame has 8 effective bytes, so the data transmission time is short, and interference or error probability is low;

8) Adopting non-destructive arbitration technology, which means that the lower priority node will stop to send messages when there're two nodes requiring to send messages synchronously;

9) Any node could break it from the CAN bus automatically when error occurred. 


\section{Hardware Design and of the Network Control System for Car Lights}

It is established a network control system through CAN bus to connect the central control unit and other two driving modules for car lights. These three CAN nodes is designed according to the generally structuralized design procedure. That's to say, all the three modules has the similar circuit which is convenient for the debugging and maintenance.

A. Topological Structure

As mentioned above, the network control system for car lights is composed of three parts: the central control unit and two other driving modules for car lights. The central control unit collects the switch information and sends the switching commands to the driving modules via CAN bus. Accordingly, the driving modules drive the car lights after receiving the CAN messages containing the switching commands. The topological structure of this network control system is depicted in Fig.1.

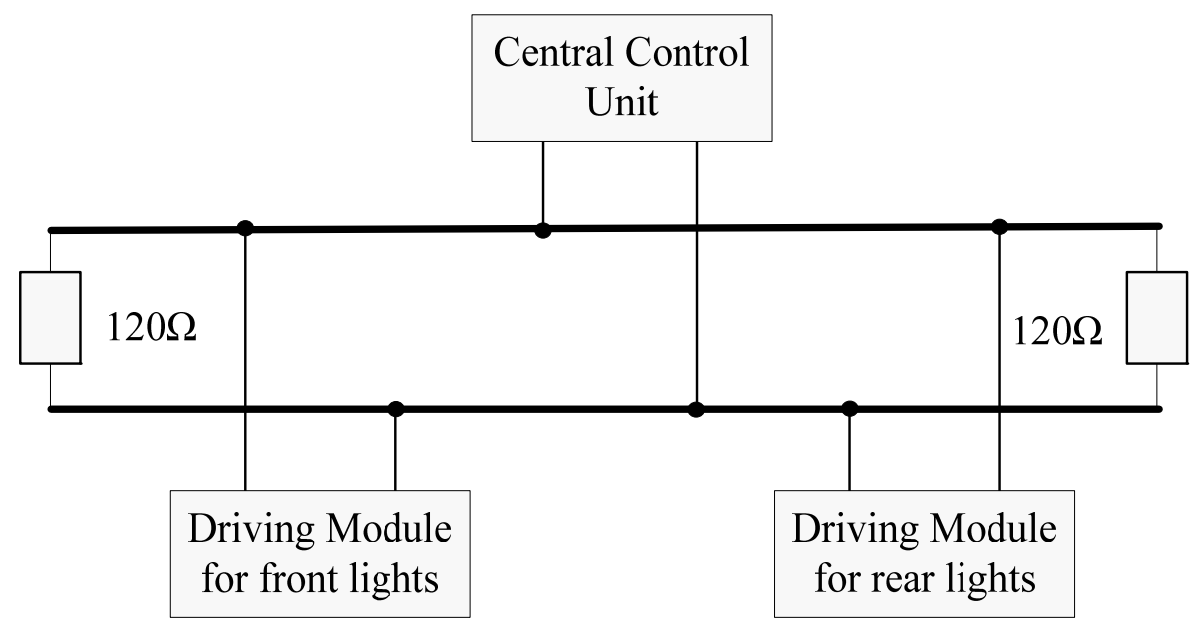

Figures1. The topological structure of the network control system

B. Design of CAN Interface

In this paper, two types of microcontroller, i.e. LPC2119 and P8xC591 have been employed to design the CAN interface. LPC2119 which is an ARM microcontroller with two internal CAN controllers has been used as the central controller of the network control system for car lights, while P8xC591 with one internal CAN controller has been used to design the driving module of car lights. Moreover, MC33889 with one internal fault tolerant low speed CAN transceiver has been used as the interface of the CAN controller and the physical layer bus. Besides, this component could supply $5.0 \mathrm{~V}$ regulated power for the microcontroller and other external components. Specifically, a general CAN interface in this scheme is shown in Fig.2.

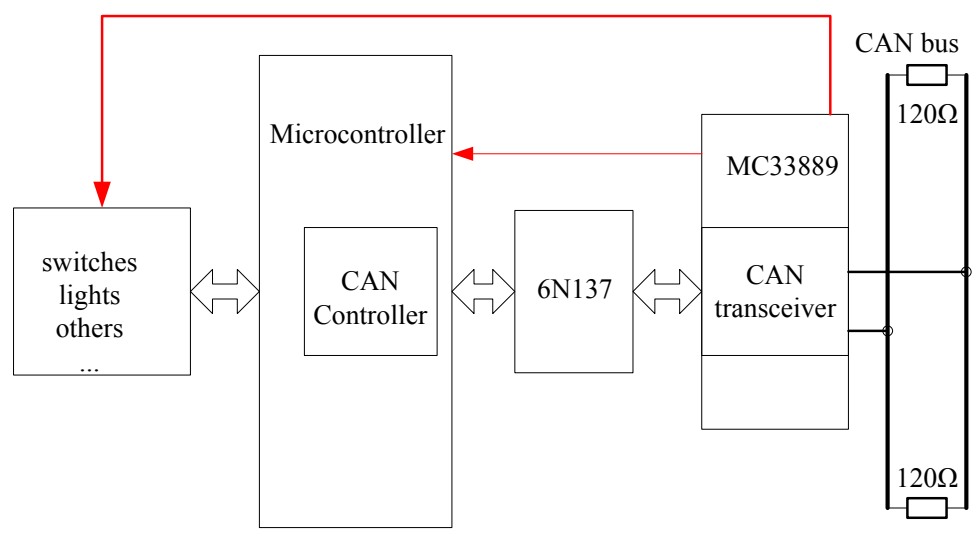

Figures2. Schematic diagram of the CAN interface

In order to improve the anti-interference capability of this CAN interface, a high speed optocoupler, i.e. 6N137 is inserted between the CAN controller and the CAN transceiver. Furthermore, a $120 \Omega$ terminal resistance is connected between CANH wire and the CANL wire to support the normal communication over the CAN bus. 


\section{C. $\quad$ Functions and Hardware Structure}

As shown in Fig.3, the central control unit and two driving modules for car lights are in charge of different electrical/electronic equipment in the car body. The central control unit has two functions, one is used as a gateway which could communicate with other network such as LIN or RS232C, the other is conducted for collecting switch information in the cab using the switch collecting IC (Integrated Circuit), i.e. MC33993. All the switch information is packed as a CAN message with a specific identifier (ID). After receiving this CAN message with the known ID, the driving modules will drive the cars lights through power switch ICs such as MC33286, MC33288 and MC33888.

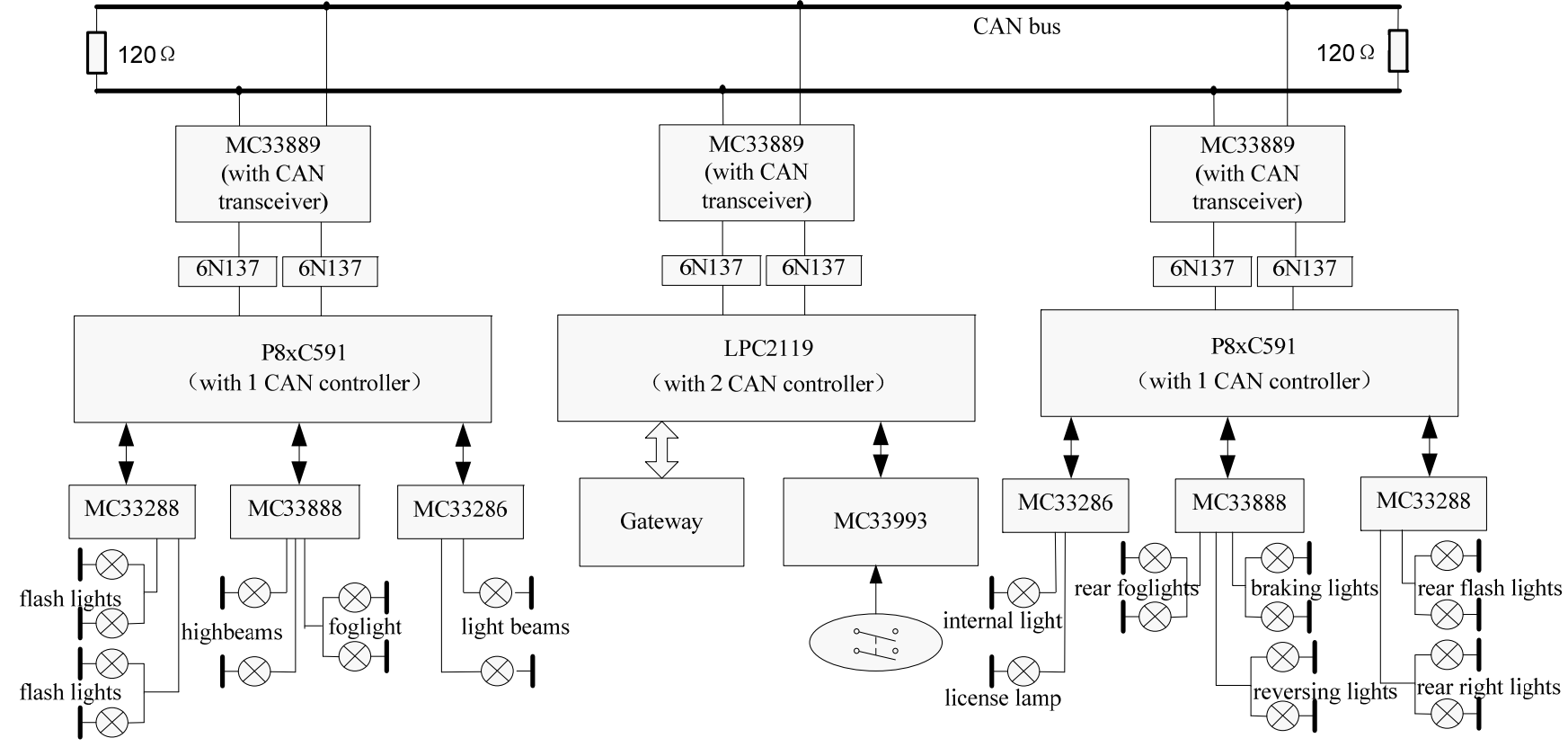

Figures3. The network control system for car lights

\section{Software Architecture and of the Network Control System for Car Lights}

In this paper, the structuralized programming method is used to design each module according to the functions of the network control system. From the robustness and reusability, modularized software is implemented. This software has been divided into three components, i.e. the main component which is responsible for the application functions, the input component which is responsible for the sensor and communication data collection, as well as the output component which is responsible for driving lamps in the car body.

Fig.4 shows the flow diagram for receiving and sending CAN message. 

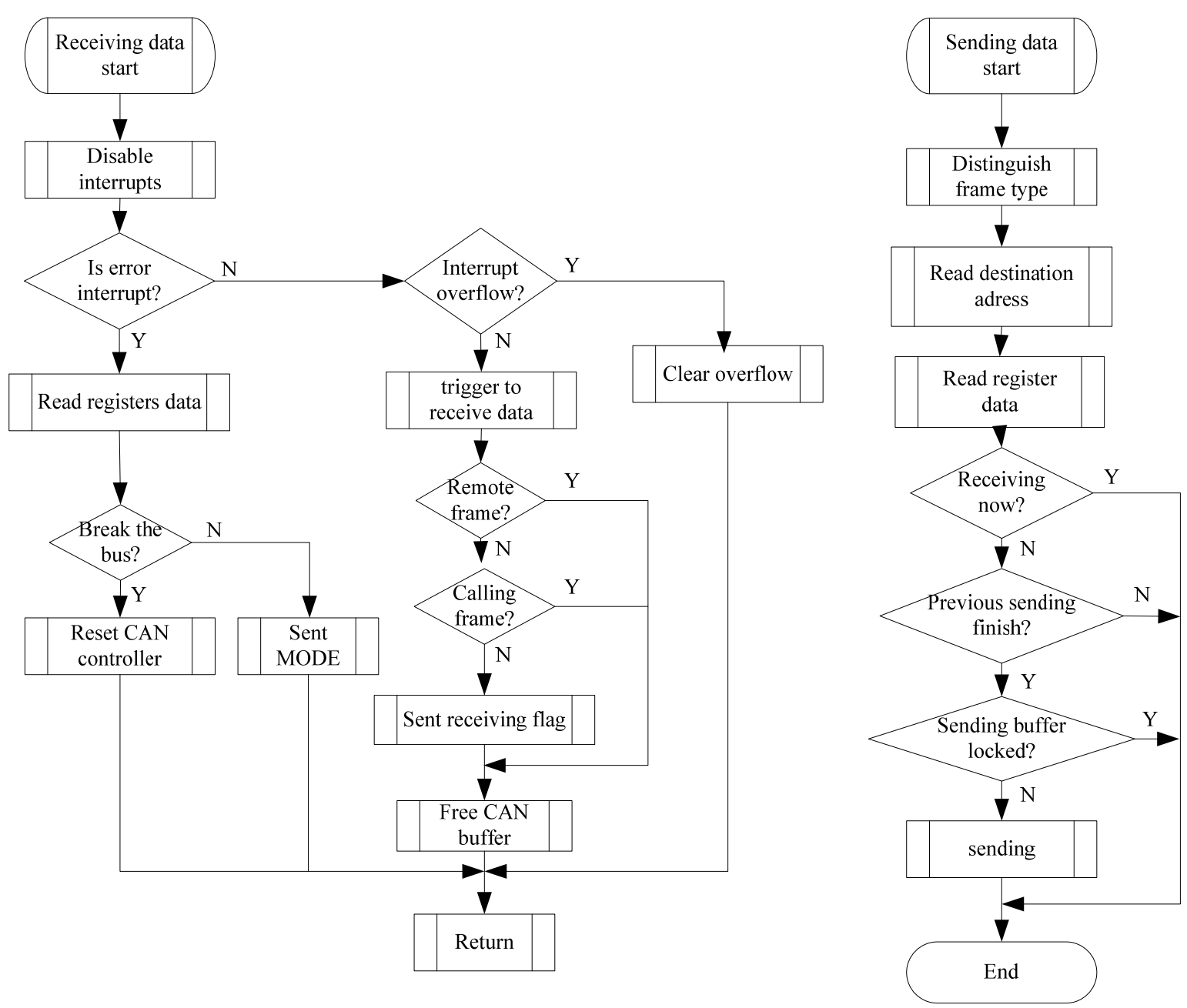

Figures4. The CAN messages receiving and sending flow diagram

\section{Conclusion}

Comparing to the traditional control mode which is point-to-point style, the network control system using CAN bus has some obvious advantages such as the simple control structure, reduced wires. Moreover, this type of network control system brings about high reliability and maintainability. Mostly importantly, the software design process of the corresponding hardware in the network control system is highly effective because the reusability of the software has also been improved. The practice proved that this system is easy to achieve and convenient for industrialization.

\section{References}

[1] Controller Area Network (CAN), CAN Specification Version 2.0, Robert Bosch GmbH (1991).

[2] SAE Standards. Application Layer, SAE J1939/71. Society of Automotive Engineers (2005).

[3] M. Zhou, X. Ao, J. Wang, Fault Diagnosis of Automobile Based on CAN Bus , Communications in Computer and Information Science, Vol. 86 (2011), p. 317-323.

[4] C. Yang, The design of distributed control system based on CAN Bus, the International Conference on Electronic and Mechanical Engineering and Information Technology, Vol. 8 (2011), p. 3956-3958.

[5] Etschberger K, CAN-based higher layer protocols and profiles, Proceedings of the 4th International CAN Conference, CAN-in-Automation, Berlin (1997). 